\title{
Significance of Mucin Stains in The Diagnosis of Carcinoma of Cervix
}

\author{
Linda Lamngaiching Changsan ${ }^{1 *}$, Pranita Medhi ${ }^{2}$ and Utpal Dutta ${ }^{1}$ \\ 'Department of Pathology, Assam Medical College and Hospital, Dibrugarh, Assam (India) \\ ${ }^{2}$ Department of Pathology, Jorhat Medical College and Hospital, Jorhat, Assam (India)
}

\begin{abstract}
Background: As many as $25-35 \%$ of carcinoma cervix without clearly defined glandular structure on histopathological examination by Hematoxylin and Eosin stains have intracellular mucin demonstrable by using mucin stains which may redefine the classification of cervical carcinomas which becomes important as mucus secreting neoplasms resembling pure squamous cell carcinomas are more aggressive and have worse prognosis than their purely squamous counterparts.

Methods: Our study conducted at Assam Medical College Dibrugarh for a period of one year. 54 cases of carcinoma cervix diagnosed on Hematoxylin and Eosin stain were subjected to mucin stains i.e. Periodic acid Schiff and Alcian blue at $\mathrm{pH} 2.5$ and the results were compared with each other.

Result: Out of the total 54 cases, 47 cases were squamous cell carcinoma (SCC, 87.03\%) which were further graded as well differentiated type (12/47) SCC, moderately differentiated (31/47) SCC, poorly differentiated (4/47) SCC and only 3 cases were adenosquamous carcinoma $(3 / 54 ; 5.5 \%)$ and adenocarcinoma $(4 / 54 ; 7.41 \%)$ by conventional H\& E stain. On subjecting to specific mucin stains, however $21.2 \%$ (10 cases) of the SCC (47 cases) contained intracellular mucin with varying percentage, along with adenocarcinoma and adenosquamous carcinomas. Revised diagnosis after application of mucins stains showed pure squamous cell carcinomas (37/54) i.e. 68.51\%, adenocarcinomas remained same at $7.41 \%(4 / 54)$ and adenosquamous carcinoma increased (13/54) to $24.07 \%$.

Conclusion: Identifying adenosquamous carcinomas after application of mucin stains which were not detectable by H\&E stained sections has sparked academic interest because of its prognostic significance as it has been found in various studies that adenosquamous carcinomas often pursue an aggressive course and are associated with worse prognosis than their pure adeno- and squamous - counterparts as most of them are poorly differentiated. So recognition of this type of carcinomas can help to predict prognosis and to plan management.
\end{abstract}

\section{Keywords: Carcinoma Cervix, Mucin, PAS \& Alcian Blue}

\section{Introduction}

Carcinoma cervix ranks the fourth most common cancer in women worldwide, and the seventh overall and its incidence varies with continents with greater percentage of the global onus found in the less developed regions, as the sensitivity of the screening tests in several low resource countries is rather poor so cervical carcinoma still continues to be the leading cause of cancer in females in developing countries and by the time the cases are detected, they tend to be at later stages of the disease. ${ }^{[1,2]}$. Multifactorial causation, potential for prevention, and the sheer threat it poses make cervical cancer an important disease for in-depth studies, as has been attempted by this study.

Squamous cell carcinomas comprise the majority of the cancers of the cervix accounting for $80 \%$ of all cervical cancers followed by adenocarcinoma which constitutes about $10-20 \%$ of cervical cancer cases. Adenosquamous and neuroendocrine carcinomas are rare cervical tumors. Routine application of stains for mucin, preferably
PAS and Combined alcian blue/PAS has shown that by classifying of carcinomas of cervix in $\mathrm{H}$ \& $\mathrm{E}$ stained section alone, many mucin secreting neoplasms escape the detection unless mucin stain is applied and shown that 20 to $30 \%$ of the cervical tumours regarded as being squamous cell carcinomas have to be reclassified either as poorly differentiated adenocarcinomas or as mixed adenocarcinomas. This reclassification is important and significance of identifying mixed tumours lies in the fact that these neoplasms run an unusually aggressive course and is associated with a much worse prognosis than their pure squamous and adeno- counterparts. ${ }^{[3]}$

The primary objective of our study is to find out prevalence of mucin secretion in cervical carcinoma diagnosed on the basis of Hematoxylin \&Eosin, (H\&E) staining by application of mucin stains.

\section{Materials and Methods}

The present study was conducted from June 2015 to May 2016 in the Department of Pathology at Assam Medical 
College, Dibrugarh during which total cases of 54 already diagnosed as cervical carcinomas on histopathological examination by Hematoxylin \&Eosin staining were studied. A detailed clinical history, clinical examination and necessary investigations were obtained in each case and gross examination of each resected mass was done in a fastidious manner and representative samples were subjected to histopathological examination. The tissues were fixed by using $10 \%$ formalin and processed according to our laboratory standard operating procedure, stained with H\&E and subsequently Periodic Acid Schiff's reagent (PAS) and Combined Alcian blue at $\mathrm{pH}$ 2.5/ PAS. Separate tissue controls were put for PAS and Alcian blue. The tumors were classified initially on $\mathrm{H} \& \mathrm{E}$ stained sections according to WHO classification ${ }^{[4]}$. After mucin stain they were re-classified into Fox classification system ${ }^{[5]}$. According to this, tumors with squamous growth pattern, keratin formation, intercellular bridges and no mucin positivity were classified into squamous cell carcinoma. The latter is subdivided into well, moderate and poorly differentiated. Tumors with acinar differentiation or widespread mucin secretion in atleast $75 \%$ of the tumor volume were labeled as adenocarcinoma. Lesions exhibiting both squamous and acinar differentiation with the minor component constituting at least one third of the tumor were considered as adenosquamous carcinoma. Squamous carcinoma exhibiting smaller quantities of mucin (not more than 30\% of the tumor volume) were diagnosed as squamous cell carcinoma with mucin secretion.

\section{Result}

From the total 54 cases, 47 were squamous cell carcinoma out of which 12 were graded well differentiated type, 31 were moderately differentiated and 4 as poorly differentiated, 3 were of adenosquamous carcinoma and 4 were adenocarcinoma. The mean age of presentation of carcinoma was 48.45 years with age groups ranging from 25 years-71 years. Most common presenting symptom was found to be abnormal vaginal bleeding/ postmenopausal bleeding $(87.04 \%$ of cases) followed by discharge per vagina, pelvic pain, post coital bleeding etc as shown in Table 1. In present study most common per-speculum/colposcopic finding was found to be cervical growth and cervical ulceration. Out of the 47 cases diagnosed as squamous cell carcinoma, 10 cases i.e. $21.2 \%$ contained intracellular mucin with varying percentage, all the adenocarcinoma and adenosquamous carcinomas contained mucin .(Fig .2Fig .4)After subjecting to mucin stains i.e. both PAS and Combined Alcian Blue at $\mathrm{pH}$ 2.5/PAS, only $68.5 \%$ turned out to belong to pure squamous cell carcinomas whereas the percentage of adenocarcinoma remained same and adenosquamous carcinoma rose to $24.07 \%$ respectively.

The statistical analysis using Chi square test with Yates correction in diagnosis of squamous cell carcinomas and adenosquamous carcinomas on H\&E stains and after subjecting them to mucin stains showed statistically significant at $\mathrm{p}<0.05$ as shown in Table 3 , however there was no statistical significance in the diagnosis of adenocarcinomas before and after doing mucin stains.

Table 1: Showing Presenting Symptoms.

\begin{tabular}{|l|c|c|}
\hline SYMPTOMS & NUMBER $(\boldsymbol{n})$ & PERCENTAGE (\%) \\
\hline Abnormal Bleeding P/V & 47 & 87.04 \\
\hline Discharge P/V & 36 & 66.67 \\
\hline Pelvic pain & 21 & 38.89 \\
\hline Post Coital Bleeding & 14 & 25.93 \\
\hline Pain during Coitus & 13 & 24.07 \\
\hline Others & 11 & 20.37 \\
\hline
\end{tabular}

Table 2: Showing Gross Findings in Per-Speculum/Colposcopic Examinations.

\begin{tabular}{|c|c|c|c|}
\hline \multicolumn{2}{|c|}{ SPECULUM COLPOSCOPIC FINDING } & NUMBER (n) & PERCENTAGE (\%) \\
\hline \multicolumn{2}{|l|}{ Cervical Ulcer } & 18 & 33.33 \\
\hline \multirow{3}{*}{ Cervical Growth } & Exophytic Growth & 21 & 38.8 \\
\hline & Infiltrative Growth & 15 & 27.7 \\
\hline & TOTAL & 54 & 100.00 \\
\hline
\end{tabular}

Table 3: Showing Revised Histopathological Diagnosis After Inclusion of Mucin Stains

\begin{tabular}{|c|c|c|c|c|c|}
\hline \multirow[b]{2}{*}{ DIAGNOSIS } & \multicolumn{2}{|c|}{ Initial diagnosis on H\&E } & \multicolumn{2}{|c|}{ After mucin stains } & \multirow[b]{2}{*}{$P$ value } \\
\hline & $\mathbf{N}$ & $\%$ & $\mathrm{n}$ & $\%$ & \\
\hline Squamous cell carcinoma & 47 & 87.04 & 37 & 68.5 & 0.036 \\
\hline Adenocarcinoma & 04 & 7.4 & 04 & 7.4 & 1.0 \\
\hline Adenosquamous carcinoma & 03 & 5.5 & 13 & 24.07 & 0.013 \\
\hline TOTAL & 54 & 100 & 54 & 100 & \\
\hline
\end{tabular}




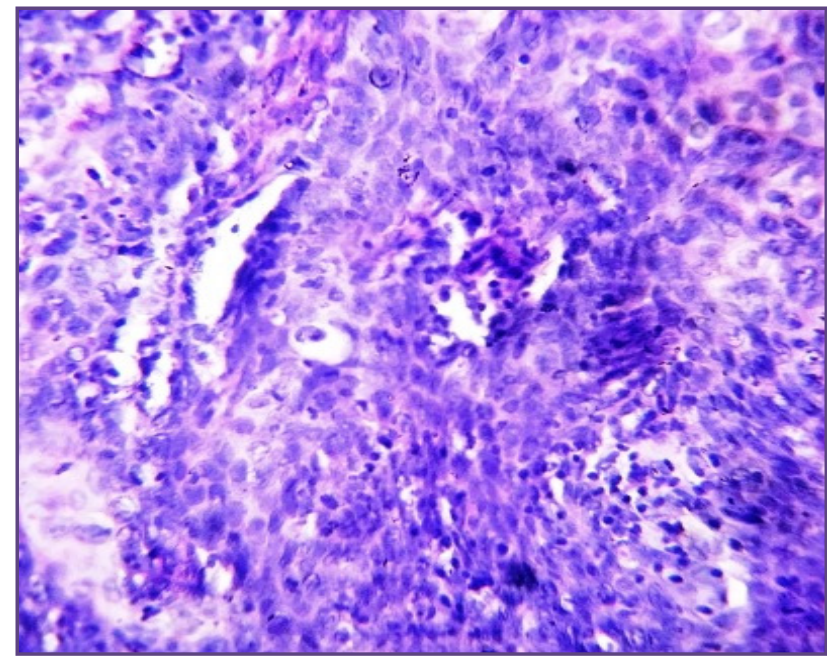

Fig. 1: Well Differentiated Squamous Cell Carcinoma with Negative PAS staining (40X).

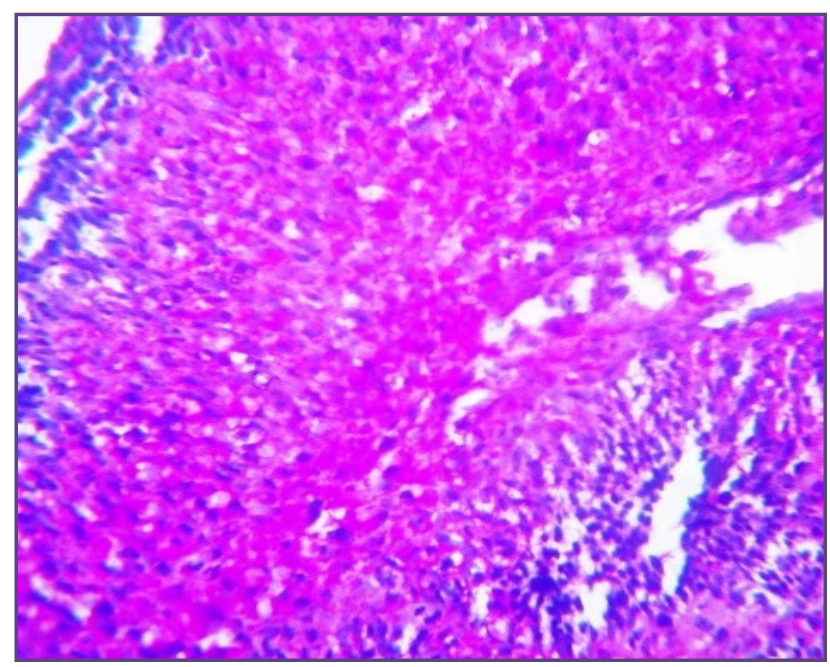

Fig. 3: Poorly Differentiated SCC with mucin content $>\mathbf{8 0} \%$ of the tumor (PAS/40X).

\section{Discussion}

Cervical cancer is a worldwide problem and ranks the fourth commonest cancer in women worldwide, and with statistics varying from country to country and from race to race. In the United States, the incidence rate has decreased during the last half century as a result of the widespread use of cervical cytologic screening programs but the same cannot be said of developing countries. Mortality due to cervical cancer is another indicator of health inequities as $86 \%$ of all deaths ${ }^{[6]}$ due to cervical cancer are in developing, low- and middle-income countries. Cervical cancer is mainly categorized into squamous cell carcinoma, adenocarcinoma and adeno-squamous carcinoma however lesion diagnosed as moderately or poorly differentiated

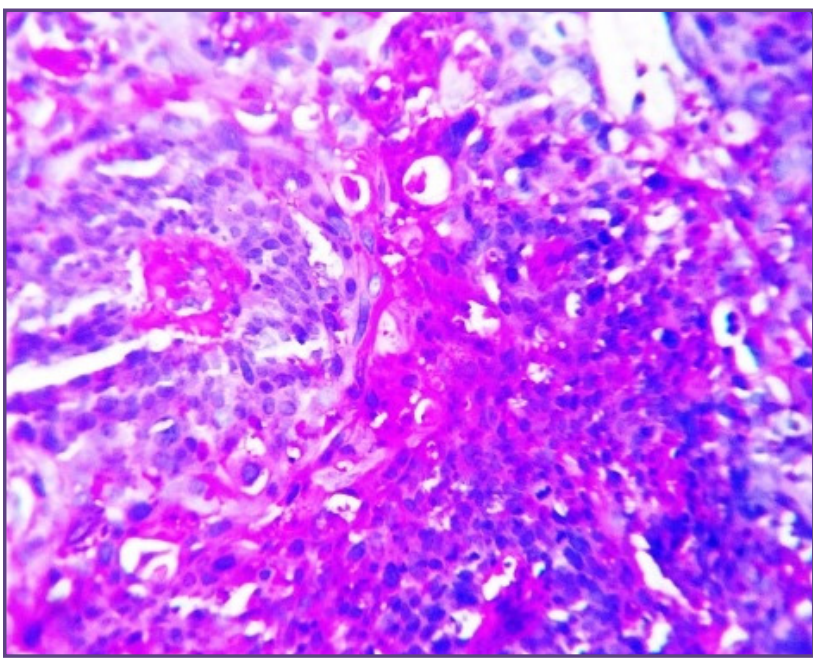

Fig. 2: Moderately differentiated SCC with PAS stained mucin in Tumor Cells.

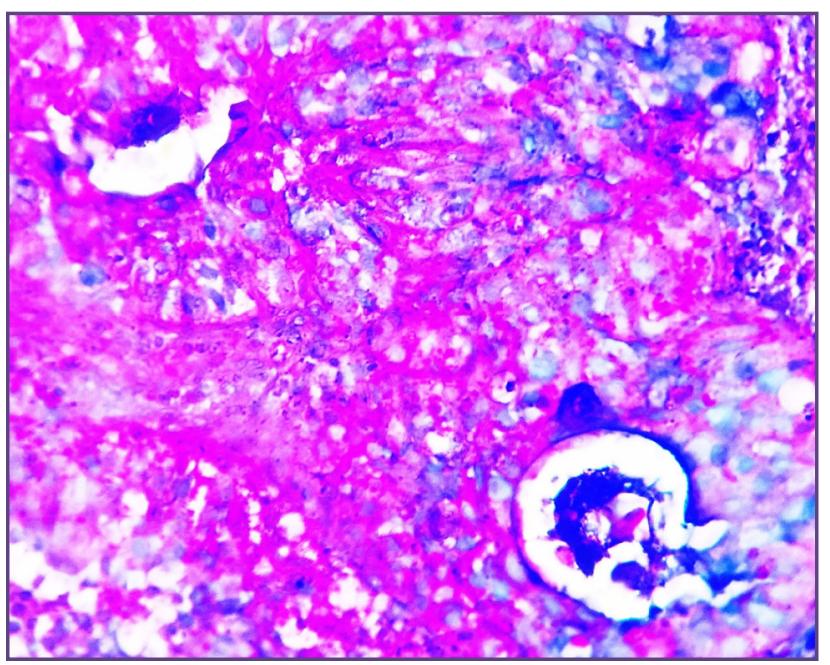

Fig. 4: Adenosquamous Carcinoma showing Combined Alcian Blue/PAS staining in the glandular component as well as other tumor cells(40X).

squamous cell carcinomas on $\mathrm{H} \& \mathrm{E}$ stain may turn out to be squamous cell carcinoma with mucin secretion, adenosquamous carcinoma or adenocarcinoma after staining with mucin stains depending upon the amount of mucin present. ${ }^{[3]}$ This gives much weight on the significance of mucin stains as a complementary to routine $\mathrm{H} \& \mathrm{E}$ staining for diagnosis of cervical carcinoma . In our study, we used two well established techniques, PAS and Combined Alcian Blue/PAS, for the detection of mucins in cervical carcinomas. Revised diagnosis after application of mucins stains showed pure squamous cell carcinomas to be $68.51 \%$, adenocarcinomas remained same at $7.41 \%$ and adenosquamous carcinoma rose to $24.07 \%$ i.e. increased to 13 cases from 3 cases. 
Our study revealed that combined AB/PAS stain preferable due to greater specificity and intensity of staining. Even though PAS also gives good results it has its drawback that, keratin also take the same stain and increases the chances of false positivity, but careful examination showed that the positivity shown by keratin was granular, it is different from block shown by mucin. The combined Alcian blue/PAS technique is a simple procedure and appears to differentiate sharply between acid mucins and neutral mucins.

Misra $V$ et al. ${ }^{[7]}$ studied biopsies of cervix uteri histochemically by using mucin stains, PAS, combined Alcian Blue/PAS, seventeen percent cases of squamous cell carcinomas showed positivity in mucin staining. Studies conducted by Preeti et al. ${ }^{[3]}$, Mathur SK et al. ${ }^{[8]}$, Keshav $P$ et al. ${ }^{[9]}$ also had similar findings .Observing most of the studies that have been done, it can be inferred that squamous cell carcinomas needs special mention as regards to mucin stain as it produce significant change in its diagnosis after application of mucin stains and diagnosis of most of the adenocarcinomas and the adenosquamous carcinomas remain unchanged before and after application of mucins. However, a much larger study over a longer period of time would accurately ascertain the worth and utility of routine mucin histochemistry as complementary to histopathological diagnosis of cervical carcinomas giving special emphasis on the poorly differentiated and moderately differentiated squamous cell carcinomas.

\section{Conclusion}

The role of histopathology as the benchmark for diagnosing invasive cervical carcinomas is undebatable and it still remains today the basic cornerstone of morphological identification of invasive cervical carcinomas but to advocate incorporation of mucin stains in the routine histological work up for diagnosis of cervical carcinomas would not be an understatement, especially poorly differentiated ones as it is of significance in determining the precise histopathological diagnosis.

\section{Acknowledgements}

We express our heartfelt gratitude to DBT Nodal center, Tezpur University for providing financial support and we are also grateful to our laboratory technicians who assist us during the course of this research

\section{Funding}

DBT Nodal Centre for Medical Colleges and Biomedical Research Institute, Tezpur University

\section{References}

1. Ferlay J, Soerjomataram I, Ervik M, Dikshit R et al. Mortality Worldwide: IARC Cancer Base No. 11.Lyon, France: International Agency for Research on Cancer; 2013.

2. Kent A. "HPV vaccination and Testing". Reviews in obstetrics and gynecology. 2010; 3 (1): 33-34.

3. Preeti, Kalhan S, Alka , Jain K et al. Should mucin histochemistry be Routinely done for carcinoma cervix. Journal of Clinical and Diagnostic Research 2010 August ; 4:2714-2719.

4. Kurman RJ, Carcangiu ML, Herrington CS et al. WHO Classification of Tumours of Female Reproductive Organs. WHO/IARC Classification of Tumours, 4th Edition, Volume 6 IARC. Lyon 2014

5. Colgan TJ, Auger M, McLaughin JR. Histopathological classification of cervical carcinoma and recognition of mucin secreting squamous carcinoma. Int J Gynaecol Pathol 1993; 12:64-69

6. Arbyn M, Castellsague X, De Sanjose S et al. Worldwide burden of cervical cancer. Ann Oncol. 2011; 22: 2675-2686.

7. V Misra, S C Gupta, A Goel et al. Re-classification of carcinoma cervix uteri by mucin histochemistry. Indian Journal of Pathology and Microbiology. 1997; 40 (4 ): 463

8. Mathur SK, Marwaha N, Arora et al. Significance of Mucin Secretion in Carcinoma of uterine cervix. India J. Pathol Microbiol. 2002; 45 (3): 261

9. Keshav P, Virendra K, Pratik C. Utility of PAS and Alcian Blue Stains in Carcinoma Cervix. International Journal of Biomedical Research 2016; 7 (8): 606.

*Corresponding author:

Dr. Linda Lamngaiching Changsan, Deptt of Pathology, Assam Medical College and Hospital, Dibrugarh 786002, Assam, India.

Phone: +91 9435510686

Email: lindachangsan@gmail.com

Financial or other Competing Interests: None. 